\title{
Immune reconstitution inflammatory syndrome in tuberculous pleurisy and ulcerative colitis: a case report
}

\author{
Federico Piffer ${ }^{1}$, Guido Levi², Giampietro Marchetti², Chiara Barbieri ${ }^{3}$ \\ ${ }^{1}$ Pulmonology Department, APSS Trento, Hospital of Arco; ${ }^{2}$ Pulmonology Unit, Spedali Civili, Brescia; ${ }^{3}$ Pulmonology \\ Unit, IRCCS Arcispedale Santa Maria Nuova, Reggio Emilia, Italy
}

\begin{abstract}
We present the case of a 46-year-old Caucasian male, affected by ulcerative colitis, who developed tuberculous pleurisy during immunosuppressive therapy; despite proper therapy, worsening of the radiological findings was observed. The case was discussed among an online group of Italian physicians and diagnosis of immune reconstitution inflammatory syndrome (IRIS) tuberculosis was established. Therapy was continued and full recovery was obtained. IRIS is a syndrome initially described during opportunistic infections in HIV infected after being placed in anti-retroviral therapy. It reveals itself through a wide variety of manifestations, including fever, lymphadenopathies, worsening of lung infiltrates, pleural or pericardial effusion, central nervous system involvement. Few data are available regarding the best therapeutic options. IRIS is an insidious and potentially serious complication of opportunistic infections in immunocompromised patients. The always wider diffusion of immunosuppressive therapies increases the number of patients at risk, therefore physicians need to be aware of the issue.
\end{abstract}

\section{Introduction}

Immune reconstitution inflammatory syndrome (IRIS) is a syndrome which develops in previously immunosuppressed

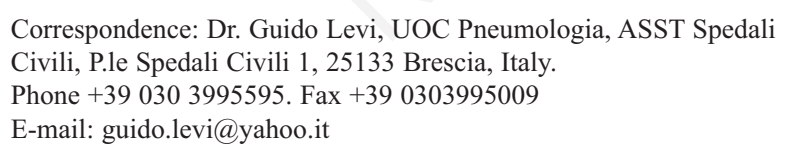

Key words: Tuberculosis; IRIS; chest ultrasound; thoracoscopy, infliximab.

Received for publication: 28 May 2019.

Accepted for publication: 24 July 2019.

${ }^{\circ}$ Copyright: the Author(s), 2019

Licensee PAGEPress, Italy

Monaldi Archives for Chest Disease 2019; 89:1103

doi: 10.4081/monaldi.2019.1103

This article is distributed under the terms of the Creative Commons Attribution Noncommercial License (by-nc 4.0) which permits any noncommercial use, distribution, and reproduction in any medium, provided the original author(s) and source are credited. patients, characterized by an overwhelming response of the immune system.

The earliest cases of IRIS were reported in 1992 among HIVinfected patients with Mycobacterium Avium complex infection after they received zidovudine monotherapy [1]; development of localized cutaneous inflammatory response to Mycobacterium Avium and fevers soon after starting zidovudine therapy was attributed to the restoration of cellular immunity to mycobacterial antigens. Since that time IRIS has been described worldwide in association with a wide range of opportunistic infections, primarily tuberculosis (TB), autoimmune diseases and malignancies $[2,3]$.

Nowadays, IRIS in HIV-TB co-infected patients is a welldescribed syndrome that results from an exaggerated immune response in the context of rapidly recovering immunity and the presence of abundant infective antigen; both adaptive and innate components of the immune system have been implicated in pathogenesis [4]. In the few studies where it has been examined, in HIV patients, IRIS is characterized by the worsening or appearance of signs, symptoms or radiographical manifestations of TB that occur after initiation of highly active anti-retroviral therapy and are not the direct result of TB treatment failure or another disease process. The symptoms and signs are often transient, but they can last many months. IRIS most often reveals itself through fever and increased or new lymphadenopathy. Commonly, the skin over the nodes can develop a dusky red inflammation and the nodes can spontaneously rupture. Pleural and pericardial effusions, ascites, psoas abscesses, cutaneous lesions, new or expanding central nervous system tuberculomata and worsening pulmonary lesions are also described [5].

The risk of TB-IRIS is higher in patients with the lowest CD4 counts at the moment of the start of anti-retroviral therapy [6]. IRIS is best described in HIV-infected patients after starting antiretroviral therapy, but it is also described in HIV uninfected patients experiencing immune recovery from other immunosuppressed states [7], including anti-tumor necrosis factor (TNF) discontinuation during biotherapy-induced TB. There is one casecontrol study that described frequency and factors associated with anti-TNF-induced TB-IRIS, matching one case of IRIS TB with two controls (that did not develop IRIS). In the French registry of opportunistic infections, severe bacterial infections, and lymphomas complicating anti-TNF $\alpha$ therapy, IRIS frequency was $7 \%$. After stopping biotherapy, paradoxical anti-TNF-associated TB worsening occurred most often in patients with disseminated TB [8]. On the therapeutic side, there is just one randomizedcontrolled trial, in HIV patients, that compares prednisone with placebo. In this trial, prednisone reduced the need for hospitalization and therapeutic procedures and hastened improvements in symptoms, performance, and quality of life [9]. 
For patients who do not respond to steroid therapy, other immunosuppressive therapies, like adalimumab and infliximab have been successfully used [10-14]. The re-start of infliximab after steroid treatment was safe in a patient with tuberculosis complicated by IRIS during anti-TNF $\alpha$ therapy for Crohn's disease [15]. The results of all these studies are presented in Table 1.

Table 1. IRIS cases in the literature: results and notes.

\begin{tabular}{|c|c|c|c|}
\hline Reference & Author & Year & Results/Topics \\
\hline 1 & French et al. & 1992 & $\begin{array}{l}42 \% \text { of patients developed a delayed-type hypersensitivity } \\
\text { response to tuberculin after zidovudine. }\end{array}$ \\
\hline 2 & French et al. & 2004 & $\begin{array}{l}\text { In some patients, suppression of HIV replication by HAART } \\
\text { causes a pathological restoration of the immune system, } \\
\text { generating the immune restoration disease (IRD). }\end{array}$ \\
\hline 3 & Meintjes et al. & 2008 & $\begin{array}{l}\text { There is an association between helper T-cell type } 1 \\
\text { expansions and TB-IRIS, but the occurrence of similar } \\
\text { expansions in non-IRIS brings into question whether } \\
\text { these are causal. }\end{array}$ \\
\hline 4 & Lai et al. & 2013 & $\begin{array}{l}\text { There are now many studies that have improved our } \\
\text { understanding of the pathogenesis of TB-IRIS and it is } \\
\text { apparent that an intricate and interconnected network of } \\
\text { both innate and adaptive immunity is involved. }\end{array}$ \\
\hline 5 & Michailidis et al. & 2005 & $\begin{array}{l}\text { 25.5\% of TB patients developed IRIS; they had significant } \\
\text { lower CD4 count and had a higher increase in CD } 4 \text { count } \\
\text { after } 3 \text { months of HAART. }\end{array}$ \\
\hline 6 & Naidoo et al. & 2012 & $\begin{array}{l}\text { More severe cases of IRIS occurred in the early treated } \\
\text { group (anti-retroviral therapy within } 4 \text { weeks of TB } \\
\text { treatment initiation). }\end{array}$ \\
\hline 7 & Sun et al. & 2009 & $\begin{array}{l}\text { IRIS has been observed in solid organ transplant recipients, } \\
\text { women during the postpartum period, neutropenic patients, } \\
\text { and tumor necrosis factor antagonist recipients. }\end{array}$ \\
\hline 8 & Rivoisy et al. & 2016 & $\begin{array}{l}14 \text { patients developed anti-TNF-associated TB-IRIS within } \\
\text { medians of } 45 \text { days after starting anti-TB therapy and } 110 \\
\text { days after the last anti-TNF infusion. The RATIO registry } \\
\text { IRIS frequency was 7\%. }\end{array}$ \\
\hline 9 & Meintjes et al. & 2010 & $\begin{array}{l}\text { The intervention group (prednisone versus placebo in IRIS } \\
\text { treatment) significantly showed an overall improvement in } \\
\text { the number of hospitalizations and outpatient therapeutic } \\
\text { procedures. }\end{array}$ \\
\hline
\end{tabular}

10 Gaube et al. 2016 A case report of cryptococcosis-related IRIS in a patient who underwent multiple relapses despite a prolonged treatment with steroids and eventually recovered with TNF-blockade therapy (adalimumab).

11 Sitapati et al. 2010 A case report of HIV-related inflammatory cerebral cryptococcoma to be treated with a recombinant human monoclonal TNF antagonist (adalimumab).

\begin{tabular}{|c|c|c|c|}
\hline 12 & Blackmore et a & 2008 & $\begin{array}{l}\text { A case report in which infliximab was used to control } \\
\text { steroid-resistant TB paradoxical reaction involving the } \\
\text { central nervous system. }\end{array}$ \\
\hline 13 & Richaud et al. & 2015 & $\begin{array}{l}\text { A case report describing a chronic debilitating } \\
\text { steroid-dependent TB-IRIS case successfully treated with } \\
\text { infliximab. }\end{array}$ \\
\hline 14 & Hsu et al. & 2016 & $\begin{array}{l}3 \text { case reports of clinical improvement temporally } \\
\text { associated with the use of infliximab for } \\
\text { steroid-unresponsive mycobacterial IRIS. }\end{array}$ \\
\hline 15 & O'Dowd et al. & 2011 & $\begin{array}{l}\text { A case report highlighting that a negative screen for latent } \\
\text { TB may be falsely reassuring in patients subsequently } \\
\text { receiving anti-TNF therapy. }\end{array}$ \\
\hline
\end{tabular}

\section{Comments}

This response could reflect the restoration of cellular immunity after zidovudine administration.

IRD management and prevention is a priority, especially in certain countries.

The defect in immune regulation responsible for TB-IRIS remains to be fully elucidated.

A better understanding of the cellular and mechanistic components involved in both the innate and the adaptive immune systems will hopefully lead to the identification of specific and sensitive biomarkers that can be used in diagnosis as well as new molecular targets for therapeutic interventions.

Patients who develop IRIS are more likely to present with disseminated TB, have a CD4 count $<100$ cells $/ \mathrm{mm}^{3}$ and have a prompt rise in CD4 count in the initial 3 months of HAART.

Initiation of anti-retroviral therapy in early stages of tuberculosis treatment resulted in significantly higher IRIS rates, longer time to resolution, and more severe cases of IRIS requiring hospitalization.

s, Non-HIV immunocompromised hosts develop IRIS when the sudden change in the dominant T helper responses to inflammation is not well balanced by anti-inflammatory responses.

After stopping biotherapy, paradoxical anti-TNF-associated TB worsening occurred most often in patients with disseminated TB.

Prednisone reduced the need for hospitalization and therapeutic procedures and hastened improvements in symptoms, performance, and quality of life.

The pathogenesis of IRIS remains partly unexplained but the increased production of pro-inflammatory cytokines such as TNF supports the use of anti-TNF therapy in IRIS, especially in those cases where high-dose steroids fail.

Systematic evaluation of the use of this class of immunomodulators for management of severe HIV-associated immune reconstitution disease is warranted.

This case illustrates a prompt, beneficial effect of infliximab therapy on the clinical and radiographic manifestations of paradoxical reaction in a patient with neurotuberculosis.

Authors suggest that TNF inhibitors can be used as salvage therapy in HIV-infected patients with steroid-dependent TB-IRIS.

Clinical improvement was temporally associated with the administration of infliximab without obvious adverse impact on immune recovery and HIV virologic control.

Observation for active TB infection in patients receiving anti-TNF therapy is vital; early reinstitution of anti-TNF therapy during anti-TB treatment is likely to be safe, and in future continuing anti-TNF $\alpha$ therapy may be preferred. 


\section{Case Report}

A 46-year-old Caucasian male was placed in immunosuppressive therapy with a high dose of steroids and infliximab for an acute exacerbation of ulcerative colitis, a disease he had been suffering from for many years.

In the days before the beginning of the immunosuppressive therapy, the patient underwent a chest X-ray, which showed no pathological findings, and a negative QuantiFERON-TB (QFT) test (AgTB1 ESAT-6 and CFP-10, CD4 T Lymphocyte $0.01 \mathrm{IU} / \mathrm{mL}$, negative if $<0.35$; AgTB2 ESAT-6 and CFP-10, CD4 and CD8 T Lymphocyte $0.01 \mathrm{IU} / \mathrm{mL}$, negative $<0.35$ ). At that time, he was in therapy with intravenous methylprednisolone $60 \mathrm{mg} / \mathrm{die}$.

After 2 months of immunosuppressive therapy, the patient was admitted to the hospital with fever and chest pain; the chest X-ray showed a small left pleural effusion. Blood tests did not show remarkable alterations: C-reactive protein was $12.6 \mathrm{mg} / \mathrm{L}$ (normal value, n.v. <5), lactate dehydrogenase 252 U/L (n.v. 136-234), fibrinogen $417 \mathrm{mg} / \mathrm{dL}$ (n.v. 170-410), albumin $3.26 \mathrm{~g} / \mathrm{dL}$ (n.v. 3.404.65). Empirical antibiotic treatment with fluoroquinolones was started (Levofloxacin $750 \mathrm{mg}$ daily per os for 10 days), without substantial improvement; the patient underwent also an ultrasound (US)-assisted thoracentesis; the procedure was completed without adverse events. The cytological sample of the fluid showed a high count of lymphocytes and no neoplastic cells. The patient underwent a High Resolution Computed Tomography (HRCT) scan that showed partially loculated left pleural effusion, with a maximum thickness of $17 \mathrm{~mm}$. In the adjacent parenchyma were reported centrilobular ground glass nodules and thickening of the septa. A bronchoscopy with broncholavage was then performed; the microbiological data on the samples resulted negative. In the hypothesis of TB pleuritis, QFT test was repeated; this second time it resulted positive (AgTB1 ESAT-6 and CFP-10, CD4 T Lympchocyte $>10 \mathrm{IU} / \mathrm{mL}$, negative if $<0.35$; AgTB2 ESAT-6 and CFP-10, CD4 and CD8 T Lymphocyte $>10 \mathrm{IU} / \mathrm{mL}$, negative if $<0.35$ ). The patient was then interrogated but he did not report any known contact with TB infected subjects.

Due to the lack of a diagnosis and the suspect of TB pleuritis, the patient was referred to medical thoracoscopy. The chest ultrasound, performed before the procedure, showed the presence of a small anechogenic pleural effusion with limited fibrin shoots and small parietal nodules. The medical thoracoscopy, performed during deep sedation on spontaneous breathing, showed the pleura infiltrated by many classical granulations and by a lower quantity of bigger nodules (Figure 1); many histological samples were collected and were sent to anatomopathological and microbiological examination. The procedure was well tolerated and the chest tube was removed $24 \mathrm{~h}$ later. The histological examination of the tissue confirmed the clinical suspect of necrotizing granulomatous pleuritis, but there was no evidence of mycobacteria in the material and no growth of mycobacteria in the microbiological samples. The colorations for fungi were negative.

One week after the thoracoscopy, the patient was then empirically placed in standard anti TB therapy with 4 drugs (isoniazid, rifampin, ethambutol and pyrazinamide) and infliximab was discontinued. After 2 months of therapy, despite a slight clinical improvement, radiological worsening was observed, with enlargement of the pleural nodules and the appearance of new, partially necrotic, alterations on parietal pleura, involving also the chest wall and para-esophageal tissue (Figure 2). At this point, the histological samples were re-evaluated with higher magnification (auramine stain) and a high number of alcohol-acid resistant bacilli was seen (Figure 1); the nucleic acid amplification test confirmed the presence of mycobacteria.

The clinical case was discussed on the Facebook Group 'Pleural Hub' [16], an Italian physicians' online group that discusses everyday complex clinical cases, mainly about pleural pathology. During the discussion, 3 main different hypotheses emerged: i) Nontubercular mycobacteria (NTMB) involvement: this was the less probable hypothesis, because of Quantiferon positivity (although some NTMB have shown the possibility of cross-reaction [17]); ii) Multi-drug resistant mycobacteria; iii) IRIS.

Molecular examination was then performed on the histological samples, showing that the mycobacteria were TB and rifampinsensitive. So, the final diagnosis was of IRIS TB, caused by the discontinuation of immunosuppressive therapy (infliximab) during TB infection. Considering the good clinical status of the patient, despite radiological worsening, anti-TB therapy was prosecuted and neither steroids nor other immunosuppressive therapy were started. The ulcerative colitis symptoms were well-controlled with both oral and rectal mesalazine daily administration.

In the following months, the patient showed progressive clinical and radiological improvement, with full clinical recovery and negative chest X-ray twelve months later. After this episode of IRIS, infliximab has been permanently discontinued.

\section{Discussion}

In our case, the suspect of IRIS was important in order to establish a correct diagnosis without further invasive procedures; the key point to establish a correct diagnosis was the molecular confirmation of the nature of the mycobacteria (TB and rifampin sensitive). The fact that the QFT test was negative three months earlier supports the hypothesis this was a primary infection during immunosuppressive therapy, even though no contacts with TB infected subjects were reported.

Another interesting point in this case is that, maybe, fluoroquinolones empirical treatment could have had a role in the false negative microbiological data at thoracoscopy. Finally, we

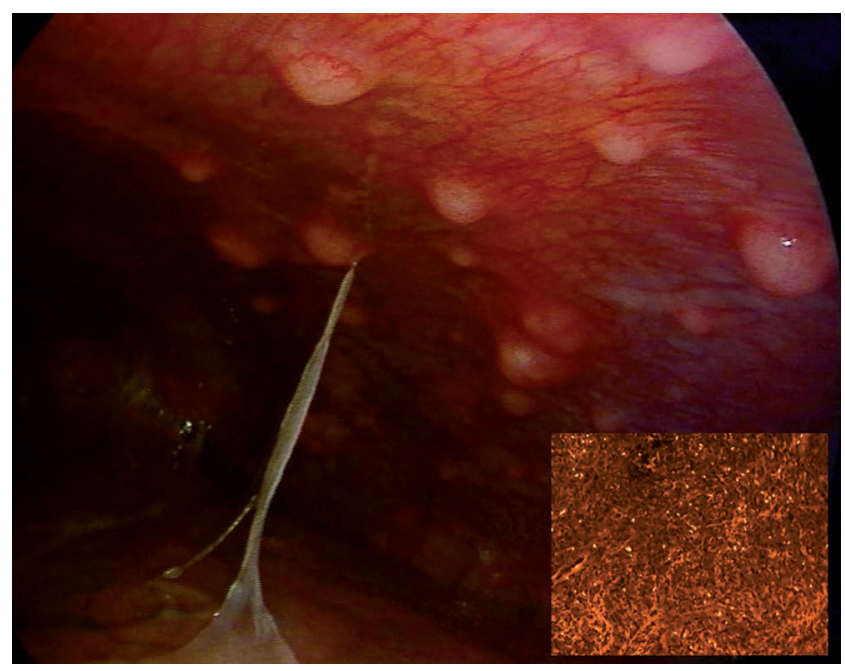

Figure 1. Thoracoscopic findings showing diffuse pleural involvement by multiple nodules; pleural biopsy (auramine stain) showing numerous tuberculosis bacilli (insert, luminescent spots). 

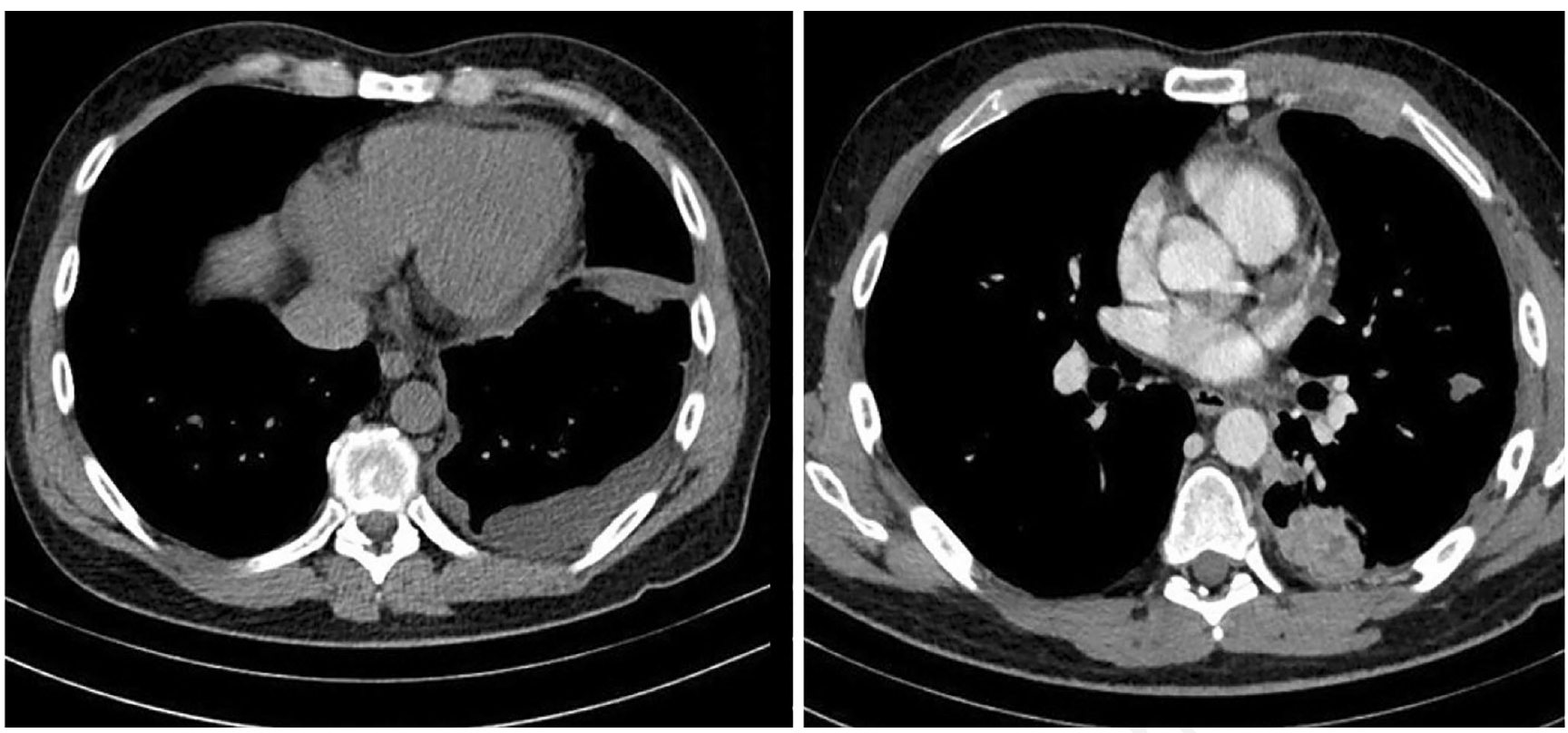

Figure 2. Comparison between the lung HRCT scan performed before the therapy (left) and the one performed after 2 months of therapy (right).

would like to point out that the sharing and the discussion among physicians played a key role in the solving of this case. In fact, as the World Health Organization (WHO) has already recommended, the correct management of multidrug resistant (MDR) and extensively drug resistant (XDR) TB infections should be ensured by the so-called "TB Consilium", a multi-specialty consultation body which includes different health care professionals. Nowadays, TB Consilia are available both at national (e.g., France, United Kingdom, Portugal, Belarus, etc.) and international level (e.g., European Respiratory Society/WHO) [18]. These extremely helpful resources are now also becoming the guiding lights in order to fight the misuse of anti-TB drugs, which is probably the main cause of increasing numbers in MDR TB infections [19]. Recently, the Global TB Network (GTN) has been launched during the last Conference of the World Association for Infectious Diseases and Immunological Disorders, which took place in Milan, Italy, in October 2018: the GTN is an online platform which aim is to collaborate with existing organizations committed to fight against TB by complementing and boosting their initiatives [20].

In conclusion, the combination of these tools and online groups on social networks, such as "Pleural Hub", could be the turning point in managing not only difficult TB cases, but most hard-tomanage clinical cases; we think this could be a rather new and important way to improve the assistance we give to our patients.

\section{Conclusions}

Immune reconstitution inflammatory syndrome remains an insidious complication of opportunistic infections in immunocompromised patients. The always wider diffusion of immunosuppressive therapies increases the number of patients at risk, therefore physicians need to be aware of the issue, even considering the potential seriousness of this syndrome.
A correct diagnosis in cases similar to the one we reported may be obtained also thanks to the cooperation among physicians; online groups could be a new and rather stimulating way to discuss complex cases.

\section{References}

1. French MA, Mallal SA, Dawkins RL. Zidovudine-induced restoration of cell-mediated immunity to mycobacteria in immunodeficient HIV-infected patients. AIDS 1992;6:1293-8.

2. French MA, Price P, Stone SF. Immune restoration disease after antiretroviral therapy. AIDS 2004;18:1615-27.

3. Meintjes G, Wilkinson KA, Rangaka MX, et al. Type 1 helper $\mathrm{T}$ cells and FoxP3-positive $\mathrm{T}$ cells in HIV-tuberculosisassociated immune reconstitution inflammatory syndrome. Am J Respir Crit Care Med 2008;178:1083-9.

4. Lai RP, Nakiwala JK, Meintjes G, et al. The immunopathogenesis of the HIV tuberculosis immune reconstitution inflammatory syndrome. Eur J Immunol 2013;43:1995-2002.

5. Michailidis C, Pozniak AL, Mandalia S, et al. Clinical characteristics of IRIS syndrome in patientswith HIV and tuberculosis. Antivir Ther 2005;10:417-22.

6. Naidoo K, Yende-Zuma N, Padayatchi N, et al. The immune reconstitution inflammatory syndrome after antiretroviral therapy initiation in patients with tuberculosis: findings from the SAPiT trial. Ann Intern Med 2012;157:313-24.

7. Sun HY, Singh N. Immune reconstitution inflammatory syndrome in non-HIV immunocompromised patients. Curr Opin Infect Dis 2009;22:394-402.

8. Rivoisy C, Tubach F, Roy C, et al. Paradoxical anti-TNFassociated TB worsening: Frequency and factors associated with IRIS. Joint Bone Spine 2016;83:173-8. 
9. Meintjes G, Wilkinson RJ, Morroni C, et al. Randomized placebo-controlled trial of prednisone for paradoxical tuberculosis-associated immune reconstitution inflammatory syndrome. AIDS 2010;24:2381-90.

10. Gaube G, De Castro N, Gueguen A, et al. Treatment with adalimumab for severe immune reconstitution inflammatory syndrome in an HIV-infected patient presenting with cryptococcal meningitis. Med Mal Infect 2016;46:154-6.

11. Sitapati AM, Kao CL, Cachay ER, et al. Treatment of HIVrelated inflammatory cerebral cryptococcoma with adalimumab. Clin Infect Dis 2010;50:e7-10.

12. Blackmore TK, Manning L, Taylor WJ, et al. Therapeutic use of infliximab in tuberculosis to control severe paradoxical reaction of the brain and lymph nodes. Clin Infect Dis 2008;47:e83-5.

13. Richaud C, Ghosn J, Amazzough K, et al. Anti-tumor necrosis factor monoclonal antibody for steroid-dependent TB-IRIS in AIDS. AIDS 2015;29:1117-9.

14. Hsu DC, Faldetta KF, Pei L, et al. A paradoxical treatment for a paradoxical condition: infliximab use in three cases of mycobacterial IRIS. Clin Infect Dis 2016;62:258-61.

15. O'Dowd C, Kewin P, Morris J, et al. Tuberculosis complicated by immune reconstitution inflammatory syndrome in a patient on anti-TNF $\alpha$ therapy for Crohn's disease. BMJ Case Rep 2011. pii: bcr0920103376. doi: 10.1136/bcr.09.2010.3376.

16. Pleural Hub Facebook Group. Accessed on: May 20, 2019. https://www.facebook.com/groups/pleural.hub/

17. Hermansen TS, Thomsen VØ, Lillebaek T, et al. Nontuberculous mycobacteria and the performance of interferon gamma release assays in Denmark. PLoS One 2014;9:e93986.

18. D'Ambrosio L, Bothamley G, Caminero Luna JA, et al. Team approach to manage difficult-to-treat TB cases: Experiences in Europe and beyond. Pulmonology 2018;24:132-41.

19. Tiberi S, Pontali E, Tadolini M, et al. Challenging MDR-TB clinical problems - The case for a new Global TB Consilium supporting the compassionate use of new anti-TB drugs. Int J Infect Dis 2019;80S:S68-72.

20. Silva DR, Rendon A, Alffenaar JW, et al. Global TB Network: working together to eliminate tuberculosis. J Bras Pneumol 2018;44:347-49. 
